\title{
Adenomatoid Odontogenic Tumor: A Case Report
}

\author{
Nithin J Jude ${ }^{1}$, Sathyanarayanan Ramanujam², Raghu Kumaravelu ${ }^{3}$, Ramya ${ }^{4}$
}

\begin{abstract}
Here, we present a case report of adenomatoid odontogenic tumor, a tumor of odontogenic epithelium in a 17-year-old young female patient who presented with a history of swelling in left side of face involving anterior maxilla which persisted for past 2 years with complaints of missing 23 and retained deciduous 62. At first, it was misdiagnosed as dentigerous cyst. Later, it was diagnosed as adenomatoid odontogenic tumor based on the biopsy report. This article focuses on various diagnostic aspects of this tumor and its surgical management. The treatment option chosen for this case was enucleation, and it is intervened under general anesthesia. On follow-up appointments, the patient reported no chance of recurrence. The conflicting views whether the lesion was neoplasm or an anomalous hamartomatous growth was also discussed. Keywords: Adenomatoid odontogenic tumor, Dentigerous cyst, Enucleation, Hamartomatous, Odontogenic cyst. Journal of Scientific Dentistry (2021): 10.5005/jp-journals-10083-0932
\end{abstract}

\section{INTRODUCTION}

A 17-year-old female presented to our institute with complaint of swelling in left side of face involving anterior maxilla for the past 2 years, which is approximately $2 \times 4 \mathrm{~cm}$ in size and also associated with missing 23 and retained deciduous 62 . The swelling is diffuse involving lateral ala of nose with expansion of buccal and palatal cortex. At first, differential diagnosis based on these clinical features were adenomatoid odontogenic tumor, dentigerous cyst, calcifying odontogenic cyst, and fibrous dysplasia. ${ }^{1}$ Then, diagnostic assessments like orthopantomogram, aspiration cytology, and biopsy were been done, which confirmed that this lesion is adenomatoid odontogenic tumor. Then, we surgically intervened it with enucleation along with removal of impacted tooth under general anesthesia, regular follow-up was done, and patient is monitored for its recurrence. AOT is a rare, slowgrowing lesion that originates from odontogenic epithelium. ${ }^{2}$ Generally, AOT do not exceed $1-3 \mathrm{~cm}$ in its greatest diameter but can be larger usually occurs within the tooth bearing area of jaws and often found in association with impacted tooth. ${ }^{3}$ AOT is also called "two-third's tumor," because two-third occur in young female, two-third occur in maxilla, two-third of cases associated with unerupted tooth, and two-third of the affected tooth is canine. ${ }^{1,3}$ Various names have been found in the literature for AOT. They were adenoameloblastoma, adenoameloblastic odontoma, aameloblastic adenomatoid tumor, epithelial tumor associated with developmental cyst, adamantinoma, pseudoadenomatous ameloblastoma, epithelioma adamantinum, and Teratomatous odontoma. $^{3-6}$ The term AOT was coined by Philipsen and Birn in 1969 and accepted by WHO in 1971..$^{2,4,6-9}$ This article, a case report of AOT, describes about clinical features, diagnostic assessments, and its surgical management in detail.

\section{Case Description}

A 17-year-old female patient reported to our department Oral and Maxillofacial surgery, Indira Gandhi Institute of Dental Sciences, Puducherry, with chief complaint of swelling in the left side of face for past 2 years. No relevant medical and dental history was obtained. On extraoral examination, inspectory findings shows facial asymmetry with diffuse swelling involving left anterior maxilla
1,2Department of Oral Surgery, Indira Gandhi Institute of Dental Sciences, Sri Balaji Vidyapeeth, Puducherry, India

${ }^{3,4}$ Department of Oral and Maxillofacial Surgery, Indira Gandhi Institute of Dental Sciences, Sri Balaji Vidyapeeth, Puducherry, India

Corresponding Author: Nithin J Jude, Department of Oral Surgery, Indira Gandhi Institute of Dental Sciences, Sri Balaji Vidyapeeth, Puducherry, India, Phone: +919787721729, e-mail: drnithinjoseph@ gmail.com

How to cite this article: Jude NJ, Ramanujam $S$, Kumaravelu R, Ramya. Adenomatoid Odontogenic Tumor: A Case Report. J Sci Dent 2021;11(1):19-22.

Source of support: Nil

Conflict of interest: None

including the lateral ala of nose with moderate obliteration of nasolabial fold (Fig. 1). On palpation, the swelling was extending 0.5 $\mathrm{cm}$ medially to the philtrum, $1.5 \mathrm{~cm}$ superiorly from the lower eyelid, $5 \mathrm{~cm}$ posteriorly from auricle, and inferiorly limited by the corner of the mouth. Swelling was firm in consistency, and mild tenderness was noted. On intraoral examination, inspectory finding shows a smooth circumscribed swelling that is approximately $2 \times 4 \mathrm{~cm}$ in size with well-defined margins in the left maxillary region obliterating the buccal vestibule. The buccal and palatal cortex is expanded with missing 23 and retained deciduous 62 (Fig. 2). On palpation, the surface of the swelling is smooth, firm in consistency with mild tenderness, extending $5 \mathrm{~cm}$ superiorly above the vestibular depth, inferiorly at the level of maxillary occlusal line, anteriorly up to right maxillary central incisor (11), and posteriorly up to left maxillary first molar (27), with displacement of adjacent teeth. At first, this lesion was differentially diagnosed as adenomatoid odontogenic tumor, dentigerous cyst, calcifying odontogenic cyst, and fibrous dysplasia. Preoperative OPG was taken later, which reveals a wellcircumscribed, unilocular, radiolucent lesion in relation to left maxilla associated with impacted 23 . A well-defined sclerotic border was found which is surrounding the radiolucent lesion. Radiograph clearly shows the juxtaposition of the impacted canine with flakes of calcification inside the lesion, and the adjacent teeth ( 22 and 24) are found to be displaced from its original position (Fig. 3). Aspiration of the lesion yielded $2 \mathrm{~mL}$ of straw-colored fluid mixed 


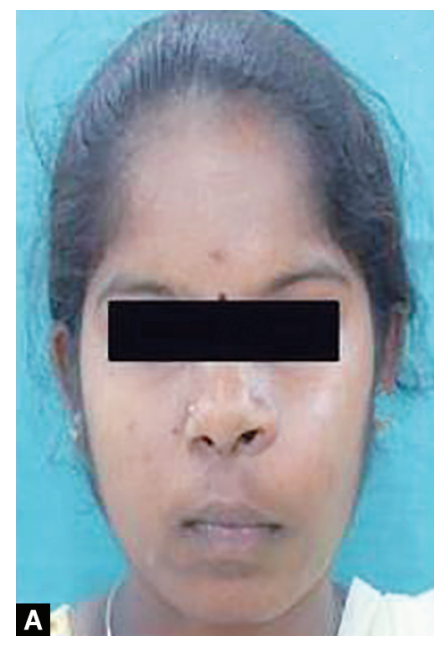

Figs $1 \mathrm{~A}$ and $\mathrm{B}$ : Front and lateral view

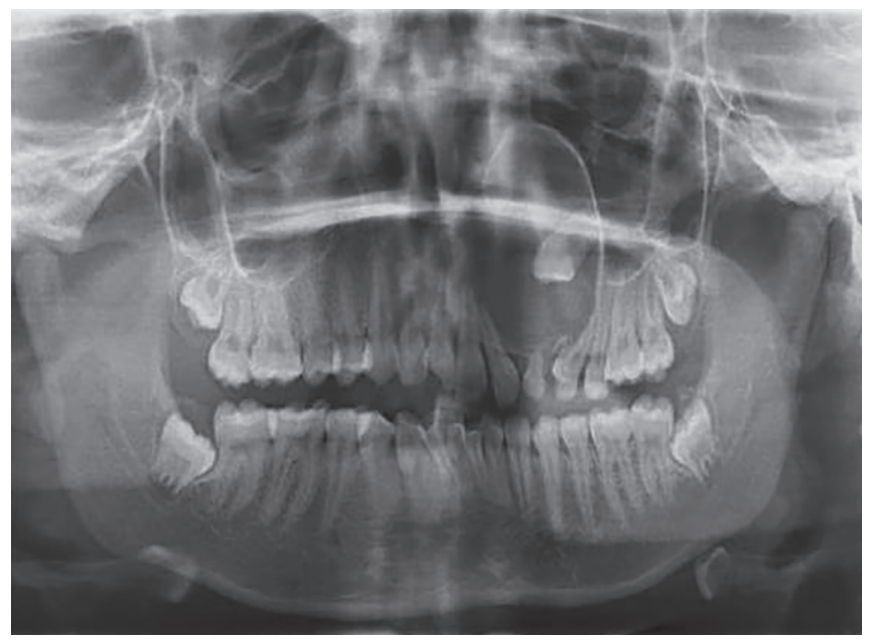

Fig. 3: OPG view

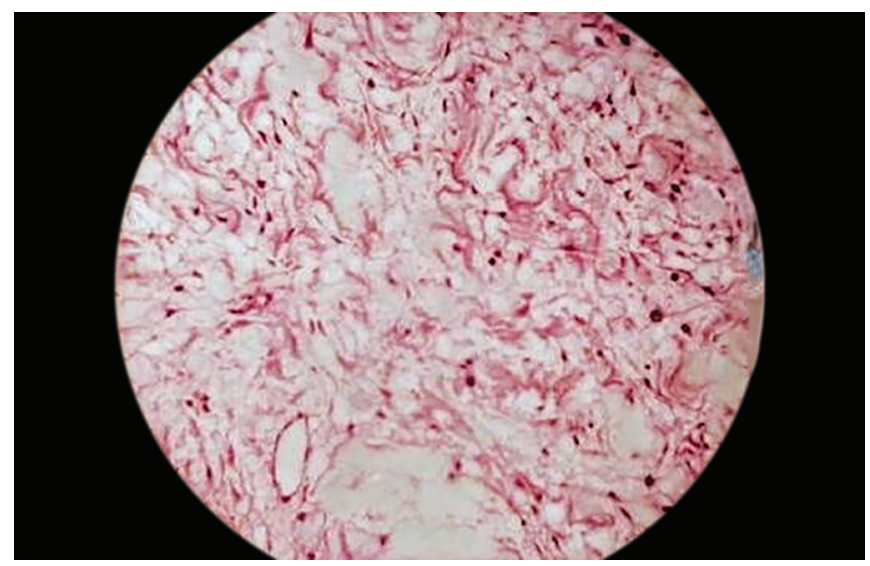

Fig. 5: Histopath view

with blood. Incisional biopsy was done under local anesthesia involving the buccal cortical plate along with cystic lining (Fig. 4). Based on these investigations, the lesion is confirmed to be AOT. Histopathological examination of this specimen microscopically reveals a well-developed connective tissue capsule surrounding a

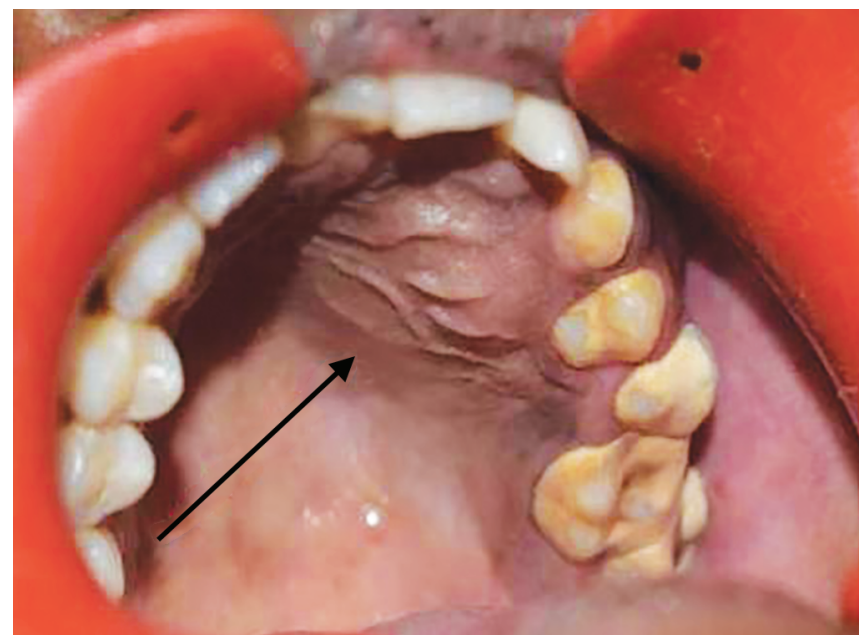

Fig. 2: Intraoral palatal view

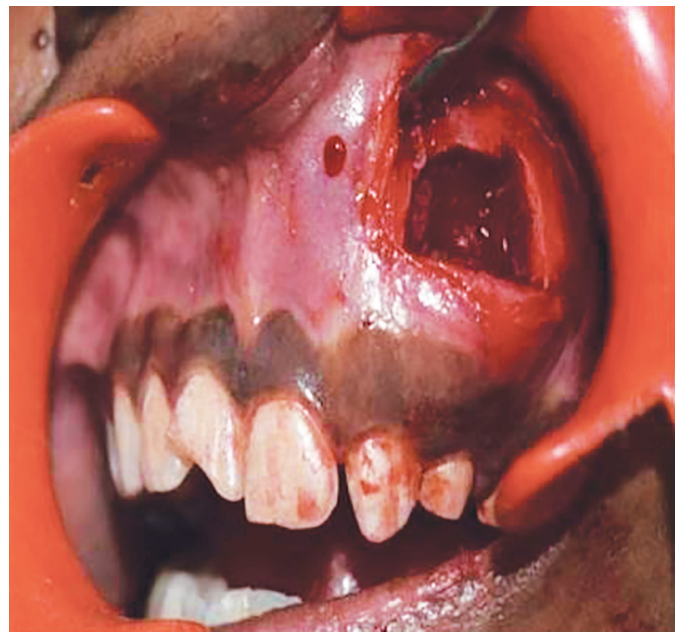

Fig. 4: Incisional biospy view

solid mass with cystic space in it. It is composed of spindle-shaped and polygonal cells forming sheets and whorled masses in a scant connective tissue stroma. Between the epithelial cells and in the center of rosette-like structure is an amorphous eosinophilic material. The characteristic duct like structure is an amorphous eosinophilic material. The characteristic duct-like structures is lined by a single row of columnar epithelial cells, the nuclei of which are polarized away from the central lumen. Dystrophic calcifications are usually encountered in AOT within the lumen (Fig. 5). Based on all these diagnostic assessment, the tumor is surgically intervened intraorally by enucleation and Curettage under general anesthesia. At first the surgical site is cleaned with betadine solution, and buccal incision was done that extends from 11 to 17. Releasing incision was given at either ends which extends into buccal sulcus, and mucoperiosteal flap is raised followed by removal of buccal cortical bone to expose the cystic lesion with constant irrigation using bone bur (Fig. 6), and now the lesion has been carefully excised along with the cystic lining, together with impacted 23 (Fig. 7). The retained 62 along with 22 was extracted, since it is associated with the lesion. Then, the cystic cavity has been irrigated with povidone iodine and saline, then packed with resorbable sponge, and then the flap is 


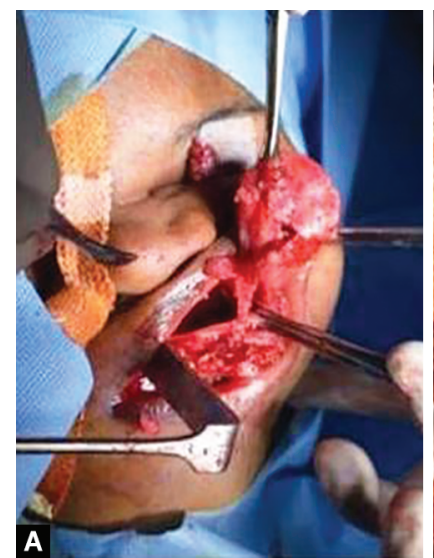

Figs $6 \mathrm{~A}$ and $\mathrm{B}$ : Intraoral surgical view

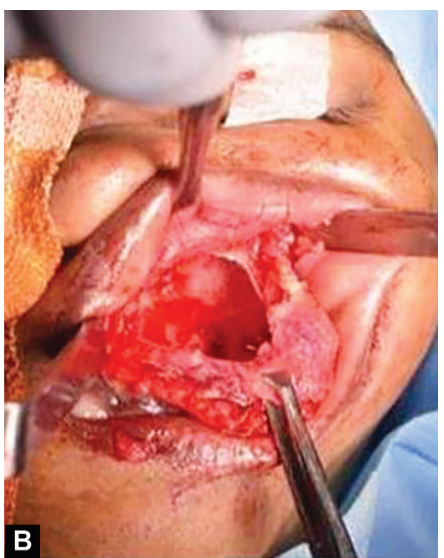

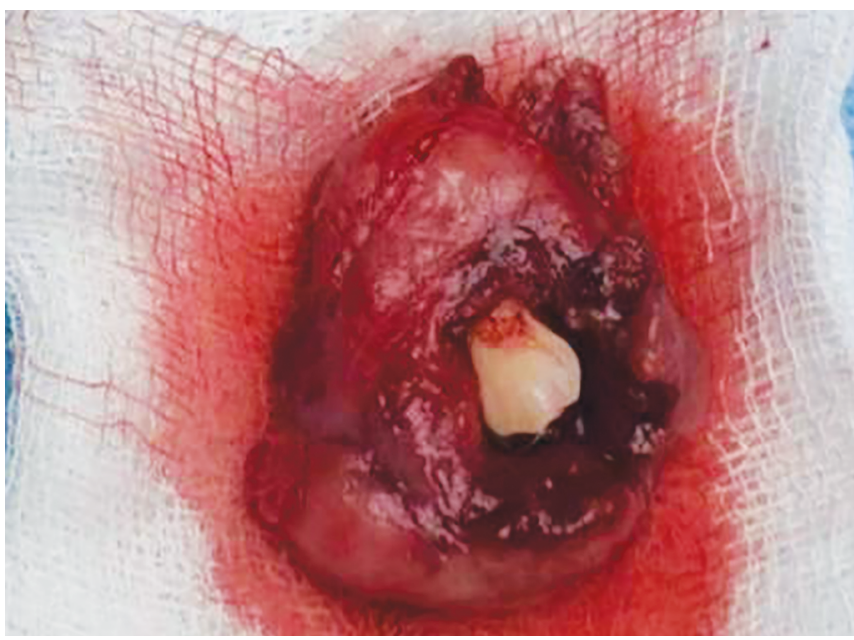

Fig. 7: Excised specimen

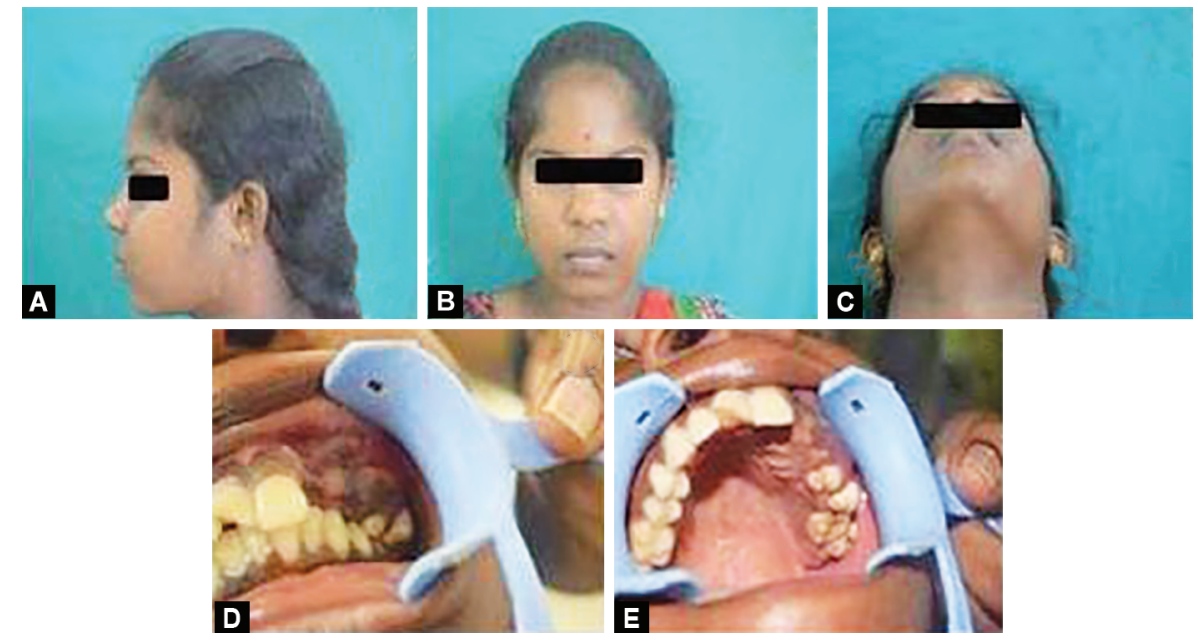

Figs $8 \mathrm{~A}$ to $\mathrm{E}$ : Postoperative view

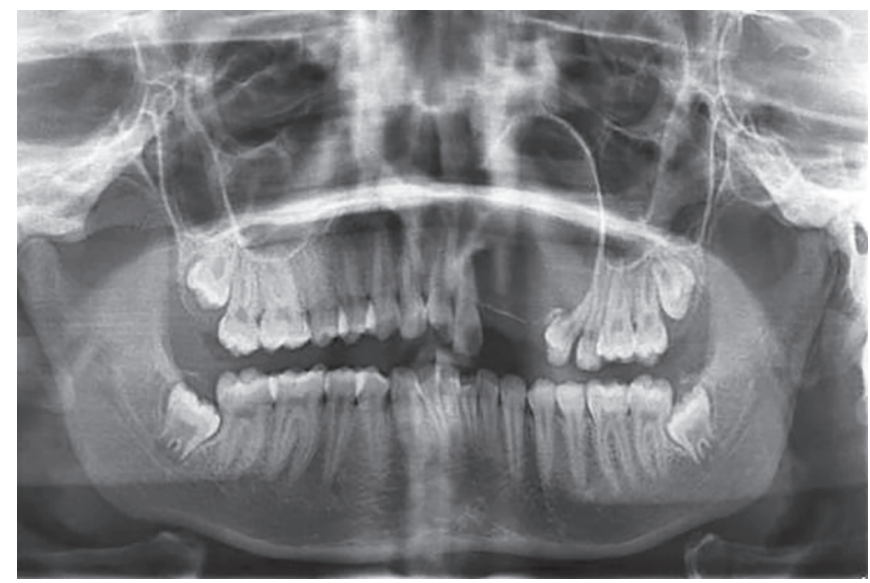

Fig. 9: Postoperative OPG

replaced back to its original position and closed with interrupted sutures. Then, the patient was maintained under regular follow-up during which healing was uneventful with almost well-formed ridge (Fig. 8), and no chance of recurrence was reported (Fig. 9).

\section{Discussion}

AOT is a rare benign tumor, since its incidence is $3-7 \%$ of all odontogenic tumor. ${ }^{1,10}$ It arises from odontogenic origin (enamel organ, epithelial remnants of malassez, epithelial lining of dentigerous cyst, remnants of dental lamina). 2,3,7,11 AOT is classified as one of the odontogenic tumors. It is a noninvasive, slow-growing lesion, mostly asymptomatic with reduced recurrence rate. It occurs in both intraosseous (96\%) and extraosseous (4\%) locations on the tooth-bearing area of both the jaws. ${ }^{4}$ It mostly affects maxilla than mandible, anteriors than posteriors, with female predilection. ${ }^{7,12}$ It is common among age-group of $10-20$ years of life (73\%) and rare above 30 years. ${ }^{10}$ AOT is mostly associated with impacted maxillary permanent canine (74\%). ${ }^{1,2,7,13}$ AOT is first recognized as a separate entity by Stafne in 1948. ${ }^{2,4,6,7,12}$ There are three variants of AOT: follicular, extrafollicular, and peripheral AOT. Follicular type (73\%) occurs intraosseously and is associated with unerupted tooth, and it is often misdiagnosed as dentigerous cyst, which is differentiated radiographically from $A O T$, as the radiolucency involves the entire length of the tooth, both crown and root unlike dentigerous cyst, where the radiolucency involves only the crown portion of the tooth and does not envelop the roots. Minute calcification 
(radiopaque structures) which present over the radiolucent area differentiated AOT from dentigerous cyst. Extrafollicular type (24\%) occurs intraosseously, and peripheral type (3\%) occurs extraosseously where both are associated with erupted tooth and found between the roots and appears as a fibroma or an epulis on the gingival soft tissue. ${ }^{2,3,5,7,13,14}$ There were two schools of thoughts, whether AOT is considered as benign neoplasm that arises from epithelial ectomesenchymal interaction or a hamartomatous growth. Histogenesis of AOT is still uncertain and sometimes categorized as hamartomatous lesion and the debate still continues. ${ }^{1}$ Some investigators consider it as being a metaplastic process rather than epithelial ectomesenchymal interaction. ${ }^{4}$ There are many treatment options, and each has its own advantages and disadvantages. Among them enucleation and curettage proved to be the best to manage AOT. 2,3,14 So, our case is surgically managed by enucleation followed by curettage with removal of impacted tooth under general anesthesia, since this is easy to access, as the lesion is well demarcated from the surrounding bone and curettage stimulates hemorrhage that results in rapid obliteration of the defect with formation of new bone. Other treatment options may sound good, but each has its own disadvantages that is comparatively more than the treatment which we preferred to be done. Say: in marsupialization (subtotal excision), the involved tooth can be preserved, and the tooth can be made to be erupted orthodontically into normal occlusion, but it has higher incidence to recur, 9,13,14 and segmental resection can be planned only in case of larger lesion $(>4 \mathrm{~cm})$ and where there is high risk of fracture. ${ }^{13,14}$ Since AOT is a rare tumor to occur, this case has been reported.

\section{ConCLUSION}

Although AOT is a rare tumor, it is a pathology and it changes one's lifestyle. So, it needs to be treated as early as possible to prevent its spread. Necessary diagnostic assessment has to be made to diagnose the pathological lesion in order to intervene the tumor surgically in a right way. Some investigators consider it as benign neoplasm, while others have categorized it as a hamartomatous malformation due to its limited size and lack of recurrence in most cases and due to its reduced growth potential. ${ }^{11}$ The most accepted treatment of choice for AOT is surgical enucleation and curettage, since no recurrence has been observed, ${ }^{2,3,9,13}$ and it has more advantages than other treatment options. The prognosis of this tumor will be favorable if the treatment has been done successfully as planned..$^{14}$ Thus, this case report of AOT summarizes the clinical features, physical examination, laboratory investigations, differential diagnosis, and its prompt treatment planning which has to be done.

\section{References}

1. Vasudevan K, Kumar S, Vijayasamundeeswari SV. Adenomatoid odontogenic tumor: an uncommon tumor. Contemp Clin Dent 2012;3(2):245-247. DOI: 10.4103/0976-237X.96837IP: 49.200. 139.122.

2. Castillejos RD, Munguia AMN, Ham GC. Adenomatoid odontogenic tumor case report and literature review. Rev Odont Mex 2015;19(3):e183-e187. DOI: 10.1016/j.rodmex.2016.02.025.

3. Singh V, Bhaskar DJ, Chandan Agali R, Kishore M, Kadtane SS, Singh $\mathrm{H}$. Adenomatoid odontogenic tumor: report of a case and review of literature. Int J Sci Study 2014;1(4):63-65.

4. Mahato B, Mandal S, Deb T, Chaudhuri K. Peripheral adenomatoid odontogenic tumor: case report and review of literature. J Oral Maxillofac Surg Med Pathol 2018. DOI: 10.1016/j.ajoms.2018.01.007.

5. Latti BR, Kalburge JV. Dentigerous cyst associated with adenomatoid odontogenic tumor (AOT) - A case report and review of literature. Med Sci 2013;1(2):44. DOI: 10.29387/ms.2013.1.2.44-47.

6. Acharya S, Goyal A, Rattan V, Vaiphei K, Bhatia SK. Dentigerous cyst or adenomatoid odontogenic tumor: clinical radiological and histopathological dilemma. Case Rep Med 2014;2014:514720. DOI: 10.1155/2014/5147200.

7. Mohamed A, Singh AS, Raubenheimer EJ, Bouckaert MMR. Adenomatoid odontogenic tumor: review of the literature and an analysis of 33 cases from South Africa. Int J Oral Maxillofac Surg 2010;39(9):843-846. DOI: 10.1016/j.ijom.2010.06.014.

8. Yadav S, Tyagi S, Kumar P, Puri N. Surgical management of adenomatoid odontogenic tumor in paediatric patient: a clinical report. J Sci Soc 2013;40(1):52-56. IP: 49.200.139.122.

9. Ide F, Kikuchi K, Kusama K. Saitama; Japan, Takashi muramatsu; Tokyo, Japan. Adenomatoid odontogenic tumor: an outdated report. AM J Orthod Dentofacial Orthop 2016;150(6):906-907. DOI: 10.1016/j. ajodo.2016.08.013.

10. Malik NA. Textbook of Oral and Maxillofacial Surgery. 4th ed., Jaypee; 2016. pp. 698-699.

11. Sivapathasundaram B. Shafer's textbook of oral pathology. 8th ed., Elsevier; 2016. pp. 113-115.

12. Dayi E, Gurbuz G, Murat Bilge O, Alif Ciftcioglu M. Adenomatoid odontogenic tumor (adenoameloblastoma). Case report and review of the literature. Am Den J 1997;42(5):315-318. DOI: 10.1111/j.18347819.1997.tb00136.x.

13. Gill DG, Schlieve TS. An aggressive anterior mandibular adenomatoid odontogenic tumor: a case report. J Dent Oral Disor 2016;2(5):. ISSN: 2572-7710.

14. Devakalavar KN, Bali A, Singh Chawla JP, Pandher PK. An atypical adenomatoid odontogenic tumor in the mandible: a report of paediatric case. Ann Pediatr Surg 2018;14:36-38. DOI: 10.1097/01. XPS.0000496989.88283.73. 